\title{
Woods versus waves: Wave attenuation through non-uniform forests under extreme conditions
}

\section{Bregje K. van Wesenbeeck ( $\nabla$ Bregje.vanWesenbeeck@deltares.nl ) \\ Deltares}

\section{Guido Wolters}

Deltares

José A. A. Antolínez

Delft University of Technology

\section{Sudarshini Kalloe}

Delft University of Technology

\section{Bas Hofland}

Delft University of Technology

\section{Wiebe de Boer}

Deltares

\section{Ceylan Çete}

Delft University of Technology

\section{Tjeerd J. Bouma}

NIOZ Royal Netherlands Institute for Sea Research, Utrecht University

\section{Research Article}

Keywords: Nature-based Solutions (NbS), flood risk, forests, levees

Posted Date: March 24th, 2021

DOI: https://doi.org/10.21203/rs.3.rs-321272/v1

License: (c) (i) This work is licensed under a Creative Commons Attribution 4.0 International License.

Read Full License 


\section{Woods versus waves: Wave attenuation through non-uniform}

forests under extreme conditions

Bregje K. van Wesenbeeck, 2, *, Guido Wolters ${ }^{1}$, José A. A. Antolínez ${ }^{1,2}$, Sudarshini Kalloe ${ }^{1,2}$, Bas Hofland ${ }^{1,2}$, Wiebe de Boer ${ }^{1}$, Ceylan Çete ${ }^{1,2}$ and Tjeerd J. Bouma ${ }^{3,4}$.

*corresponding author. E-mail address: Bregje.vanWesenbeeck@deltares.nl, B.K.vanWesenbeeck@tudelft.nl

1. Unit for Marine and Coastal Systems, Deltares, $2600 \mathrm{MH}$ Delft, The Netherlands

2. Department of Hydraulic Engineering, Delft University of Technology, 2600 GA Delft, The Netherlands

3. NIOZ Royal Netherlands Institute for Sea Research, Department of Estuarine and Delta Systems, and Utrecht University, P.O. Box 140, 4400 AC Yerseke, The Netherlands

4. Department of Physical Geography, Utrecht University, P.O. Box 80.115, 3508 TC Utrecht

\section{Abstract}

Worldwide, communities are facing increasing flood risk, due to more frequent and intense hazards and rising exposure through more people living along coastlines and in flood plains. Nature-based Solutions (NbS), such as mangroves, and riparian forests, offer huge potential for adaptation and risk reduction. The capacity of trees and forests to attenuate waves and mitigate storm damages receives massive attention, especially after extreme storm events. However, application of forests in flood mitigation strategies remains limited to date, due to lack of real-scale measurements on the performance under extreme conditions. Experiments executed in a large-scale flume with a willow forest to dissipate waves show that trees are hardly damaged and strongly reduce wave and run-up heights, even when maximum wave heights are up to 2.5 meters. It was observed for the first time that the surface area of the tree canopy is most relevant for wave attenuation, but that the very flexible leaves hardly add to effectiveness. Overall, the study shows that forests can play a significant role in reducing wave heights and run-up under extreme conditions. Currently, this potential is hardly used but may result in considerable cost savings in levee designs. 


\section{Introduction}

Vegetated foreshores, such as marshes and mangroves, are promoted globally for their capacities in reducing impacts of waves, winds and surges ${ }^{1-6}$. Besides along coastlines there is also potential for reducing wave heights and run-up in rivers and lakes by floodplain vegetation and riparian forests 7,8 . Although the capacity of trees to reduce hydrodynamic energy is intuitive, their effectiveness under more extreme events is not well substantiated with quantitative evidence. Numerical models generally oversimplify vegetation by representing it as rigid cylinders ${ }^{9,10}$. Laboratory-flume studies with scaled forests result in parameterized bulk drag values, but these cannot be readily used for realscale extreme situations. Small-scale experiments suffer from scale-effects, such as flow around natural trees representing a multiscale problem, with a large range of length scales of leaves, branches and stems of varying flexibility and higher influence of wall friction from the flume affecting drag coefficients ${ }^{11,12}$.

Previous field and laboratory-flume measurements on wave attenuation over grassy vegetated foreshores and plants show that energy dissipation depends on incident wave energy, ambient water depth, and the (vertical) structure and flexibility of vegetation ${ }^{13-17}$. Field studies included significant wave heights up to around $0.6 \mathrm{~m}^{15,18}$ with extremes up to $1.0 \mathrm{~m}$. Möller et al. (2014) carried out flume experiments exposing real flexible grassy vegetation to maximum wave heights of $0.9 \mathrm{~m}$. In contrast, for forests no such large-scale quantitative evidence exists for storm conditions. Current field observations represent relatively mild conditions with significant wave heights in the range of 0.1 to $0.5 \mathrm{~m}^{19-21}$. To obtain a quantitative understanding of wave-attenuation capacity of forests under more extreme conditions, we ran real-scale flume tests with various water levels and significant wave heights up to $1.5 \mathrm{~m}$, using both intact and defoliated 15 years old willows (Salix alba) trees.

\section{Results}

\section{Experimental set up}

We constructed a real-scale willow forest in a wave flume of $300 \mathrm{~m}$ long, $5.0 \mathrm{~m}$ wide and $9.5 \mathrm{~m}$ deep. The forest existed of 32 willow trees that were placed in 16 rows of 2 to build a 40-meter long forest on an 85-meter-long platform (Figure 1). The pollard willows (Salix alba) existed of stems that were 15 years old and branches that were 3 years old since the last cutting. Willows were placed with their roots (in a clod) in the sandy base of the platform and fixated by applying a concrete layer of $20 \mathrm{~cm}$ as bed. At the back of the forest a concrete levee slope was present (Figure 1). 
A
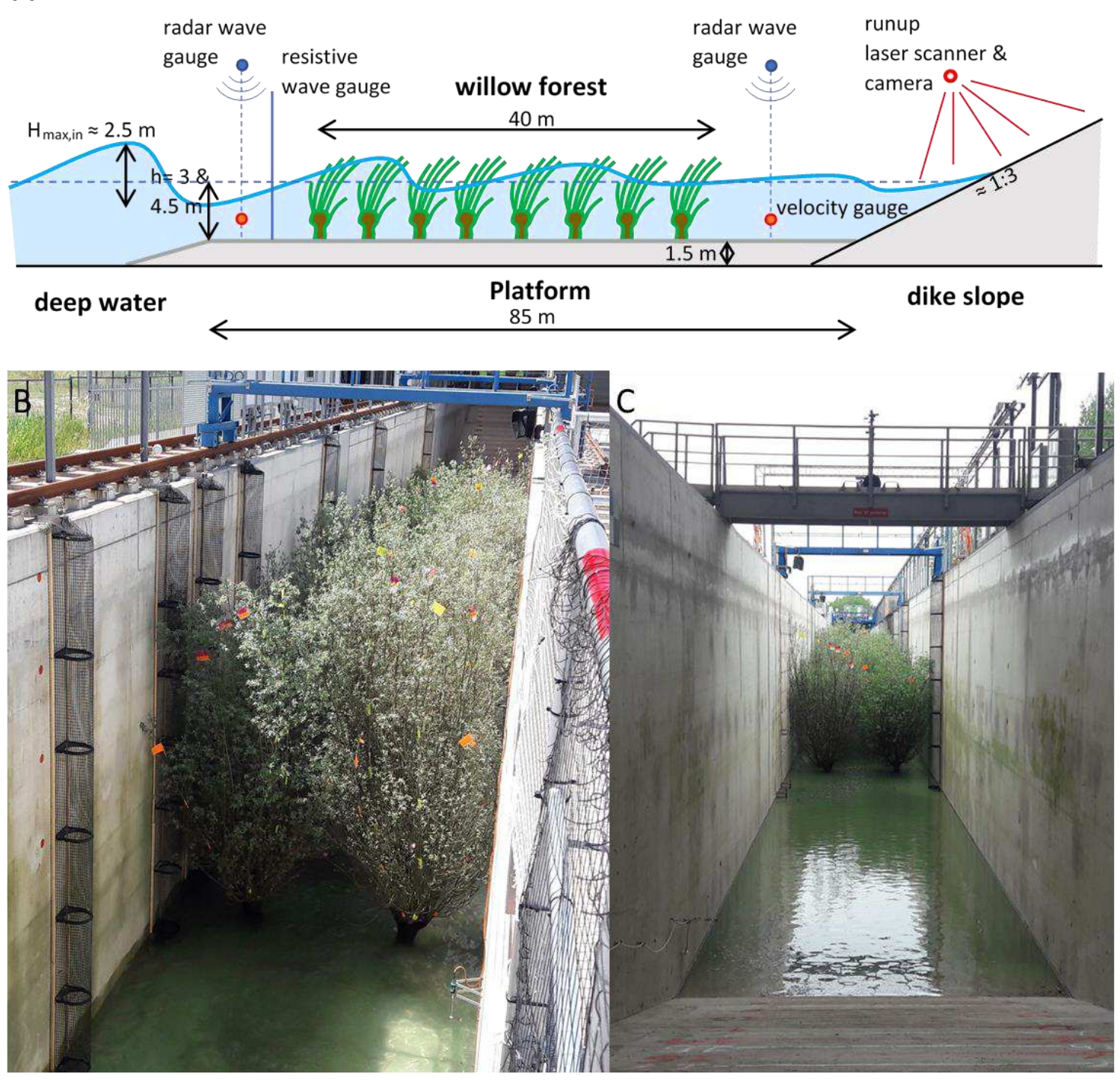

Figure 1. A. Setup of experiments inside the Delta Flume with the most important instruments, where WHM = wave gauge (resistance type), Radac = nonintrusive radar wave gauge, $E M S=$ electromagnetic velocity sensor, $B$. Front top view of willow forest, $C$. view from the back slope.

Wave attenuation by the willow forest and associated run-up on the slope were measured for different water levels in the forest ( $h=3.0$ and $4.5 \mathrm{~m}$ ), significant wave heights at the start of the forest at WHM $6\left(\mathrm{H}_{\mathrm{m} 0}=0.2 \mathrm{~m}-1.5 \mathrm{~m}\right)$ and different steepness $(\mathrm{Sop}=0.02-0.06)$. All tests were performed with a JONSWAP wave spectrum and a duration of 500 waves per test to allow for a proper statistical analysis of the wave characteristics. Test series on willows with leaves, without leaves, with a thinned branch density and, as control, without any willows (bare platform) were executed (Table 1, for all tests see Supplementary Information). Wave characteristics were measured in front of the platform, in front of the forest and behind the forest using resistance wave gauges and radar wave gauges. Wave run-up on the slope was measured using cameras, a laser scanner and visual recordings. 
Table 1. Summary of the tested hydrodynamic conditions for the different tree forest configurations (series). Test series 1 is omitted due to low water depths. All values are based on WHM6 in front of the forest with significant wave height ( $\mathrm{HmO}, \mathrm{i})$, maximum wave height (Hmax), wave period (Tp), water depth ( $h$ ) and wave steepness (Sop)

\begin{tabular}{|l|l|l|l|l|l|l|}
\hline & series & $\mathbf{H}_{\operatorname{mo,i}}(\mathbf{m})$ & $\mathbf{H}_{\max }(\mathbf{m})$ & $\mathbf{T}_{\mathbf{p}}(\mathbf{s})$ & $\mathbf{h}(\mathbf{m})$ & $\mathbf{S}_{\text {op }}(-)$ \\
\hline willow with leaves and full canopy & $\mathbf{2}$ & $0.43-0.97$ & $0.74-1.75$ & $2.84-5.57$ & $3.00-4.50$ & $0.02-0.05$ \\
\hline willow without leaves and full canopy & $\mathbf{3}$ & $0.43-1.41$ & $0.72-2.45$ & $2.84-6.85$ & $3.00-4.50$ & $0.02-0.06$ \\
\hline $\begin{array}{l}\text { willow without leaves with reduced } \\
\text { canopy }\end{array}$ & $\mathbf{4}$ & $0.43-1.44$ & $0.78-2.52$ & $2.84-6.85$ & $3.00-4.50$ & $0.02-0.06$ \\
\hline no willows & $\mathbf{5}$ & $0.17-1.43$ & $0.26-2.51$ & $1.78-6.85$ & $0.60-4.50$ & $0.03-0.05$ \\
\hline
\end{tabular}

\section{Reduction in wave height and run-up through the forest}

The wave attenuation effect of the forest was represented as the measured transmitted wave height behind the willow forest (i.e., with leaves, without leaves, reduced branch density), in reference to the case with bare platform (without willows) (Equation 1 in Methods). Plotting the wave attenuation as function of the significant wave height, $\mathrm{H}_{\mathrm{m} 0 \text {,i, }}$, shows that for constant water depth the wave damping increases as a function of wave height (Fig. 2A). The maximum wave attenuation by the willow forest is approximately $22 \%$ over 40 meters. Maximum attenuation is found for the willow forest with leaves and full canopy (Series 2), as could be expected based on the amount of frontal surface areas around the water line. Wave damping with leaves is $1.5 \%$ to $4 \%$ (percent point) higher than for a canopy without leaves (i.e., approximately $20 \%$ over 40 meters). Wave attenuation with full canopy density but without leaves is $3 \%$ to $7 \%$ (percent point) larger than with a reduced canopy density (i.e., approximately $15 \%$ over 40 meters). Wave attenuation was found to be strongly dependent on water depth. Attenuation for a water depth of $3 \mathrm{~m}$ is significantly larger than for $4.5 \mathrm{~m}$. As effects of the bottom are already accounted for in our calculation method for wave attenuation, this likely is explained by the fact that the strongest wave damping occurs when the water depth is around the middle of the canopy height (above the trunk), where the tree has most frontal surface area. The loss of biomass during different test series was relatively small. Limited breaking of stems or branches was recorded throughout repeated extreme tests, including average wave heights of 1.5 meter and maximum wave heights of 2.5 meter. It seems that the extreme flexibility of the willow branches limits the amount of actual breaking. 

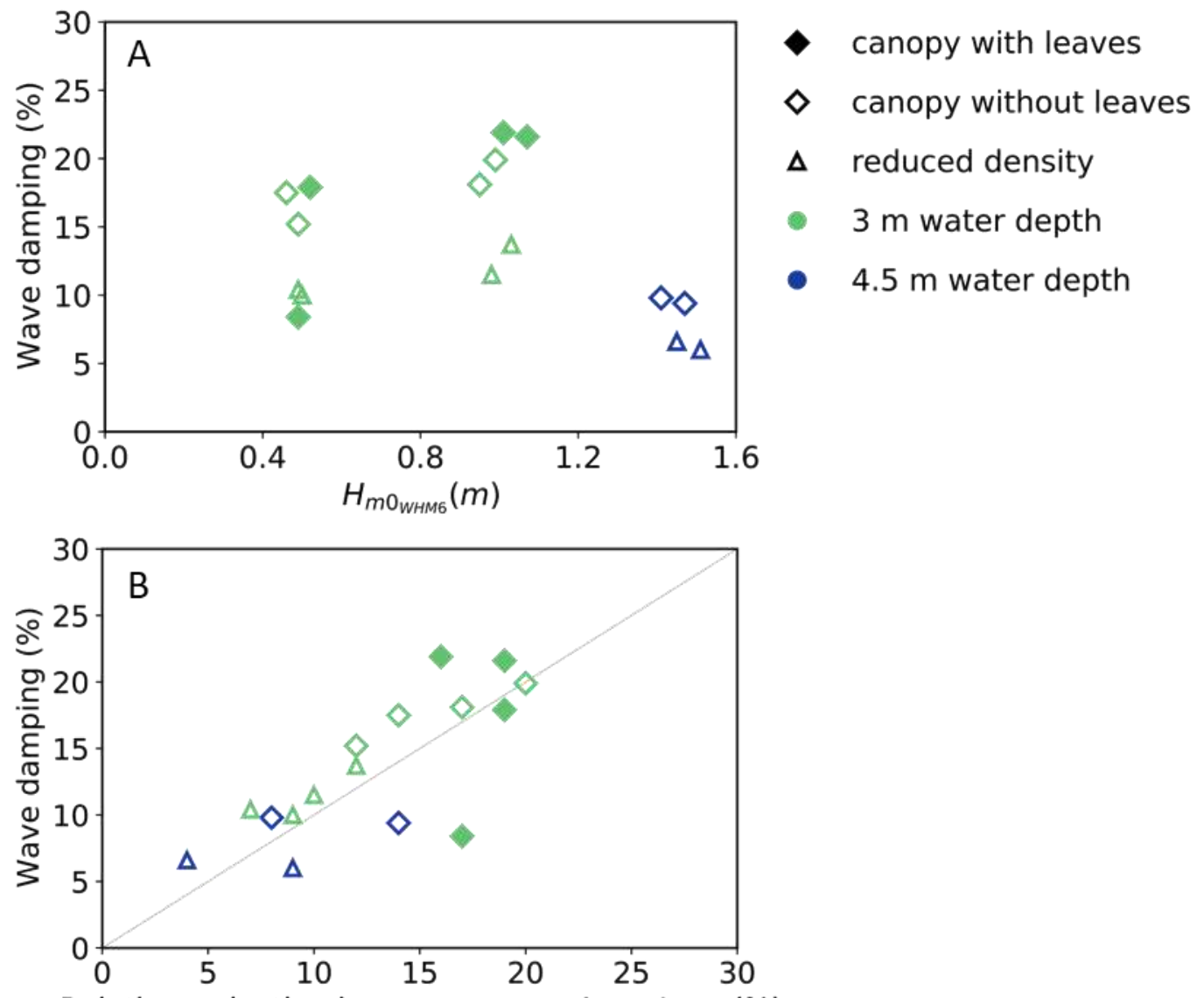

Relative reduction in wave run-up, $\Delta z_{2} \% / z_{2} \%(\%)$

Figure 2. A. Relation between measured wave attenuation (\%) and incoming significant wave height (Hm0) at WHM6. Markers represent the different willow forest configurations ( $2=$ with leaves, $3=$ without leaves, $4=$ reduced density branches), and the colors show water levels (green $=3 \mathrm{~m}$ and blue $=4.5 \mathrm{~m}$ ), B. Relation between relative reduction in wave run-up on the slope and the wave attenuation through the forest.

The wave attenuation by the willows was also assessed using the measured (reduction in) wave runup on the dike (Figure 2B). Plotting the relative reduction in wave run-up height $\left(z_{2 \%}\right.$,no_willows $-z_{2} \%$,willows $) / z_{2 \%}$,no_willows $)$ against the wave attenuation reveals a clear linear relation, and thus a similar magnitude of the reduction effect (i.e., up to 20\%). Also, the observed trends are similar, such as an increase in run-up reduction for increasing wave heights and lower run-up reduction for reduced canopy density. Note that in most cases the wave attenuation based on the wave run-up is somewhat lower than the wave attenuation based on the incident wave height. The lower outliers in the graph correspond to tests with a wave height of $\mathrm{H}_{\mathrm{m} 0, \mathrm{i}}=1.5 \mathrm{~m}$, the largest wave heights. For these tests the wave damping based on the wave run-up is somewhat larger than the damping based on the incident wave height.

Implications of measurements for wave-vegetation modelling Until now, uneven biomass distribution over the vertical and differences between stems, branches and leaves is limitedly included in numerical models. Furthermore, trees are mostly assumed to behave as a rigid material under extreme hydraulic forces. We utilized the new measurements, to 
validate models representing wave attenuation by vegetation. For this, the spectral wave model SWAN (Simulating Waves Nearshore) ${ }^{22}$ was used. This model was used in similar studies on wave attenuation over vegetated foreshores ${ }^{8,10,14}$ and is frequently used in engineering practice. Suzuki et al. (2011) implemented the effects of vegetation in SWAN based on the phase-averaged wave energy dissipation model due to rigid stems for irregular waves ${ }^{23,24}$. The vegetation model is based on bulk wave dissipation (integrated over all wave frequencies), which is dependent on the incoming wave energy, the water depth and the vertical structure of the vegetation (Equation 2 in Methods).

In previous work the vegetation is described by a single branch diameter $\left(b_{v} ; m\right)$ and density $\left(N_{v} ; m^{-2}\right)$ per vertical elevation level ${ }^{10,24}$. However, plants have different branches of different sizes and densities. Therefore, this was rewritten into a single parameter $\mathrm{f}_{\mathrm{i}}(\mathrm{z})\left(\mathrm{m}^{2} / \mathrm{m}^{3}\right)$, which described the total frontal area per unit volume, instead of $b_{v} N_{v}\left(m / m^{2}\right)$, that is generally used. This parameter is determined for the present trees by counting all branches at breast level, measuring their diameter and then applying the branching model of Jarvela (2004) ${ }^{25}$ (Figure 3a). Only branches larger than 3 $\mathrm{mm}$ were considered.

For vegetation-wave models, especially the value of the bulk drag coefficient $\left(\tilde{C}_{D}\right)$ has been subject to debate. For flexible vegetation the value of this factor is reduced compared to the value for rigid cylinders because flexible vegetation moves with the flow, which results in less drag force experienced by the vegetation ${ }^{26}$. The $\tilde{C}_{D}$ parameter relies on complex physics (e.g., skin friction, pressure differences, swaying of vegetation), which in turn depend on the vegetation properties in relation to the hydraulic conditions ${ }^{14}$. Therefore, instead of determining the $\tilde{C}_{D}$ values a-priori, several studies have attempted to calibrate the $\tilde{C}_{D}$ values to measurements and relate them to the Reynolds number ${ }^{27-30}$ or the Keulegan-Carpenter number KC ${ }^{24,31-33}$.

Here, the $\tilde{C}_{D}$ versus the KC number, $\hat{u}_{s} T_{m} / d$ was obtained for the present tests (Figure $3 \mathrm{~b}$ ). The KC number that is used here is based on the spatial weighted average of branch diameter and velocity, and the orbital motion based on $\mathrm{H}_{\mathrm{s}}$ and wave period $\mathrm{T}_{\mathrm{m}}$. Values for low $\mathrm{KC}$ numbers are close to the relation by Keulegan and Carpenter (1958) ${ }^{34}$ for a single rigid cylinder, as at these KC numbers branches do not bend much. For larger $\mathrm{KC}$ numbers the drag coefficient is decreasing, which might be due to increasing motion of the branches that reduces the relative flow velocity. With our measurements we extended $\mathrm{Cd}$ values for larger KC numbers, showing that $\mathrm{Cd}$ values for larger KC numbers are rather constant. Also, Cd values for large KC number from our experiments are considerably lower than values from previous small-scale experiments (Jadhav et al., 2013; He et al., 2019), which might be due to wall friction that is incorporated in the Cd values derived from smallscale experiments or to swaying of tree branches which was observed in our experiments. Mendez 
and Losada (2004) show low Cd for low KC numbers as these were small scale experiments with very flexible vegetation. For rigid mangrove mimics, Maza et al., (2019) ${ }^{11}$ measured values of $\tilde{C}_{D}$ between 0.5 and 1.5 at scale 1:6. However, such relations did not exist yet for woody vegetation with a complex vertical structure under extreme conditions on realistic scales (i.e., a wide range of $\mathrm{Re}$ and $\mathrm{KC}$ numbers).

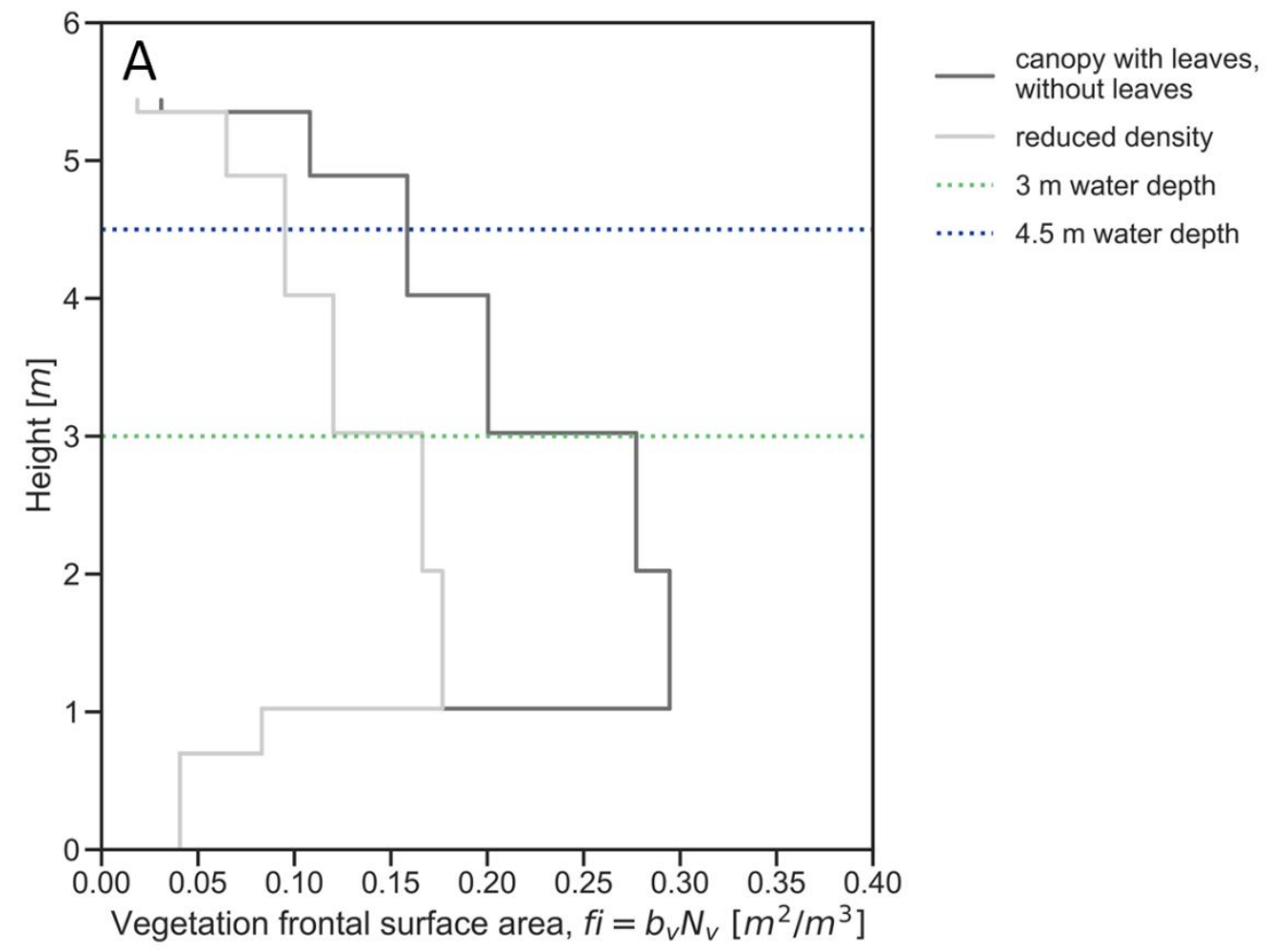




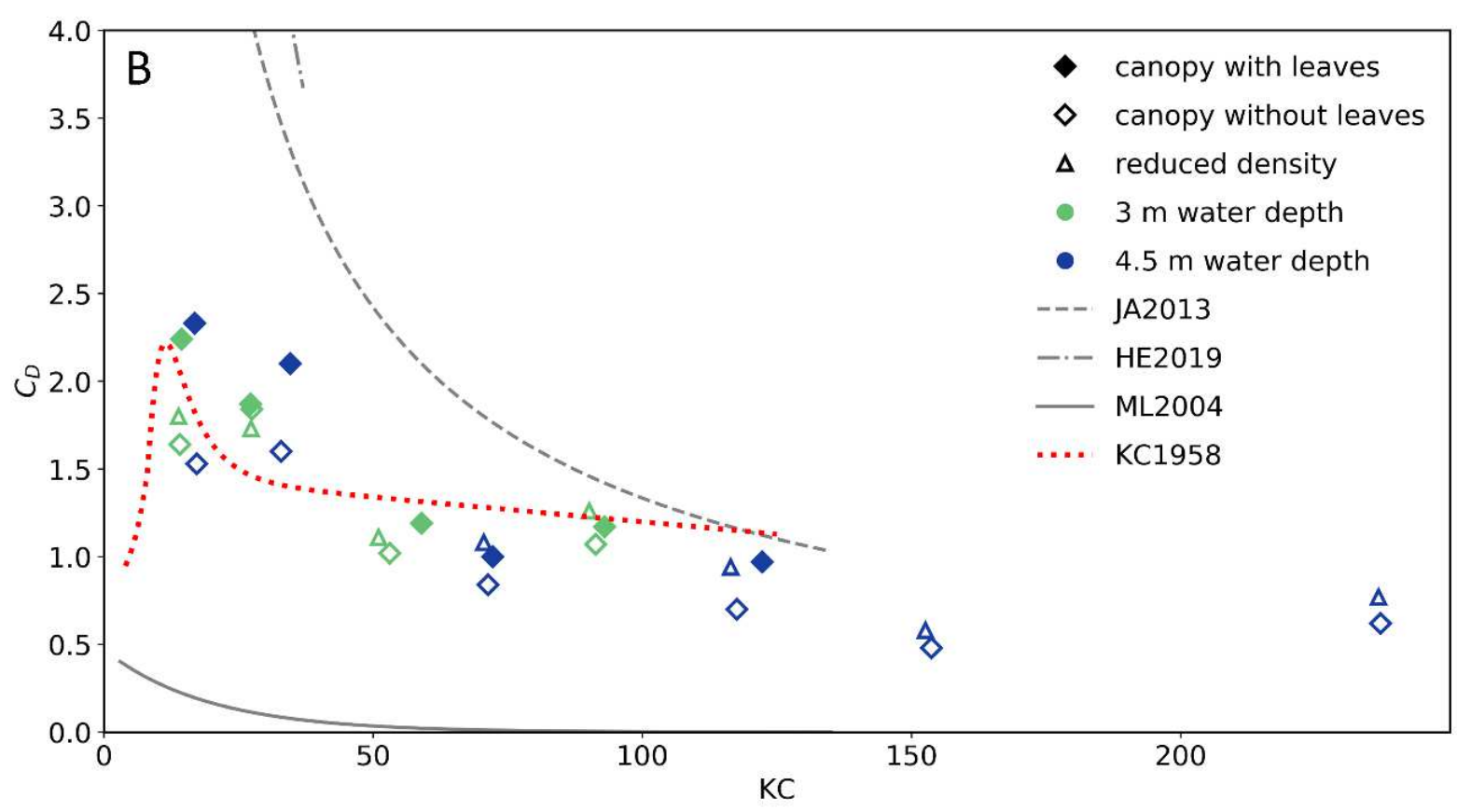

Figure 3. A. Frontal surface area of willow schematization (based in biomass schematization) for test series with full biomass with and without leaves ( 2 and 3 ) and with half of the branches removed (4), B. The KC-CD relation for all test series $(2+3+4)$ and the comparison with the relations by He et al. (2019) (HE2019), Jadhav et al. (2013) (JA2013), Mendez and Losada (2004) (ML2004), Keulegan and Carpenter (1958) (KC1958). Steady flow would be a straight line equaling Cd =1.2.

\section{Discussion}

The present real-scale tests added crucial measurements on the wave attenuation and run-up reduction by forests under extreme conditions and clearly illustrate that floodplain and mangrove forests can contribute significantly to flood safety. This constitutes yet another step towards largescale implementation of vegetation and levee combinations, or so-called hybrid solutions ${ }^{35}$. These solutions have already been promoted as the way forward under climate change and rising sea levels, as they are considered more adaptive and resilient to uncertainty in environmental boundary conditions ${ }^{8,36}$. In addition, combinations of vegetated foreshores and levees offer multiple benefits next to flood risk mitigation, such as recreation, biodiversity and fisheries ${ }^{36}$. Proper inclusion of natural landscapes in flood risk reduction and coastal protection schemes can potentially result in a larger appreciation of natural landscapes, hence lead to more effective conservation of coastal and riverine landscapes that currently often lose out against economic developments.

Caution should be taken to promote trees as a generic solution for mitigation of extreme hazards as localized studies are always required. With respect to the measurements, the present study deals with emergent vegetation only and the usually observed regime shift with vegetation submergence does not take place and effects of changes in wave profiles or currents are limited (Jacobsen et al., 2019). We also focus on wind waves, as infra-gravity waves are typically not present in situations with riparian vegetation where willow grows. For mangroves growing along more open coasts, infra-gravity waves may play a role. Effects of more diverse forests, such as mangrove forests with different age stands 
and biomass distribution, have not been explored yet. However, considering the importance of biomass distribution which was demonstrated by these experiments, more diverse forests may lead to unforeseen results. Although tests with more tree species are obviously desirable, current experiment generated unique first quantitative insights in the wave attenuation capacity of mature trees that can directly be used for modelling and optimizing foreshore management. For application in the field, wave damping is just one of many design aspects that needs to be considered in the safety assessment of a willow forest. Other aspects such as maintenance, uprooting of trees, and damage due to illness or fire are worth exploring. Nevertheless, the first examples of levee foreshore combinations are emerging in the field ${ }^{37}$ and likely more are yet to come.

\section{Methods}

Experimental set-up. Large scale experiments were conducted on living pollard willow trees in a 300 $\mathrm{m}$ long, $5 \mathrm{~m}$ wide and $9.5 \mathrm{~m}$ deep wave flume (Delta Flume) located at Deltares Research Institute in Delft, the Netherlands. In total 32 pollard willow trees (Salix alba) were placed with their roots in a sandy base of a $0.2 \mathrm{~m}$ high and $85 \mathrm{~m}$ long platform. Trees were fixated by applying a concrete layer of $20 \mathrm{~cm}$. The platform acted like a foreshore which led the incoming waves to shoal in front of the forest. These trees were placed in 16 rows of 2 and formed a $40 \mathrm{~m}$ long forest behind which a concrete levee slope (of 1:3 in the lower part and 1:3.6 in the upper part) was present.

Wave conditions and vegetation configurations. The experiments consisted of a series of tests for extreme hydraulic conditions, with varying significant wave heights $\left(H_{m 0}=0.2-1.5 \mathrm{~m}\right)$, wave periods $\left(T_{m 0}=1.8-6.8 \mathrm{~s}\right)$, wave lengths $\left(L_{p}=12 \mathrm{~m}-44 \mathrm{~m}\right)$ and water depths in the forest $(h=3.0 \mathrm{~m}$ and $4.5 \mathrm{~m})$. The tests were conducted on three different vegetation states: full canopy with leaves (series 2), full canopy without leaves (series 3) and reduced canopy density without leaves (Series 4). A series of control tests (series 5, without vegetation) were performed in order to quantify the effects of the forest on the waves. The irregular waves were generated with a JONSWAP wave spectrum and a duration of 500 waves per test to allow for a proper statistical analysis of the wave characteristics.

Wave measurements. The incoming wave height was measured based on a wave gauge (WHM6) and velocity sensor in front of the forest. WHM6 was validated for all test series using the radar measurements (RADAC1) in front of the forest. Wave attenuation by the vegetation during each test is defined as the wave height reduction relative to the incoming wave height, which is obtained from the difference between the wave energy spectra measured with and without vegetation:

Wave attenuation $=\frac{H_{m 0, \text { no willows }}-H_{m 0, \text { willows }}}{H_{m 0, \text { no willows }}}$

(Equation 1) 
With: $\mathrm{H}_{\mathrm{mo}}$ the significant wave height behind the forest

This method to assess wave attenuation proved most reliable, since it allowed us to exclude effects of wave reflection and damping effects of the platform (which resulted in additional wave attenuation of 2 to $18 \%$ ). Also, wave run-up on the slope was measured using cameras, a laser scanner and visual recordings.

Vegetation measurements. Experimental research and field studies on plants (either cultivated or wild), including the collection of plant material, was complied with relevant institutional, national, and international guidelines and legislation. Willows were harvested from private lands where they had been growing for 15 years. They contained stems that were 15 years old and branches that were 3 years old since the last cutting. The branches of the willow trees were categorized into 3 classes based on their diameter at breast height $(D B H)$, namely class $1(D B H>50 \mathrm{~mm})$, class $2(20<D B H \leq$ $50 \mathrm{~mm}$ ) and class $3(D B H<20 \mathrm{~mm})$ (see Figure S4 in Supplementary Information). Relevant tree data was gathered manually, among which: the total number of branches per class for each tree at breast height, the $D B H$ and branch length for 340 branches, and detailed sketches of 9 primary branches. The total amount of leaves were gathered and dried (at $60^{\circ} \mathrm{C}$ for $48 \mathrm{hrs}$ ) in order to measure the total dry weight. Also, bending tests were conducted to obtain the flexural rigidity for different segments of the primary branches.

Frontal surface area distribution. The frontal surface area distribution over the vertical was determined for vegetation configurations with and without leaves. The total frontal surface area of the leaves was estimated by the product of the measured total dry weight $(38 \mathrm{~kg})$ and a specific leaf area of $145 \mathrm{~cm} 2 / \mathrm{gram}^{38}$, resulting in a value of $1 \mathrm{~m} 2 / \mathrm{m} 3$ for the leaves. However, under wave loads, leaves bend, leading to a more stream-wised position. Assuming that leaves were situated with the smallest frontal area facing the stream, the specific leaf area becomes $1 \mathrm{~cm} 2 / \mathrm{gram}$ (considering a leaf thickness of $0.34 \mathrm{~mm}$, leaf width of $20 \mathrm{~mm}$ and dry weight of $0.07 \mathrm{gram}$ ), which corresponds to a projected surface area of approximately $0.005 \mathrm{~m} 2 / \mathrm{m} 3$. This shows that the contribution of the leaves to the total frontal surface area is limited.

The tapering form of the branches and the occurrence of side branches in the upper layers $(i>2)$, lead to varying frontal surface areas (equivalent to $N_{i} \cdot b_{v, i}$ ) over the vertical. We used a branching method to estimate the total frontal surface area of each tree $\left(f_{\text {total }}\right)$. This method was developed by Jarvela (2004) and originates from the Strahlers ordering scheme. This ordering scheme characterizes branch orders (i.e., the conjunction of two branches of order " $m$ " result in an order " $m+1$ " branch, starting with the smallest branches, which are assigned to order $m=1$ ). It requires only a few initial 
tree parameters (such as the average diameter of the smallest branches, $d_{\min }$, and the average diameters of the highest order, $d_{\text {high }}$, in this case $\left.D B H\right)$ to estimate total frontal area of a tree $\left(f_{\text {total }}\right)$ by using branching factors $\left(R_{B}, R_{D}, R_{L}\right)$ between subsequent branch orders. A more detailed description of the steps is given in the work of Jarvela (2004). A factor of 0.5 was applied to the resulting frontal area per order, to account for the frontal surface area of a cone shaped branch instead of cylinders. Although this method predicts the total frontal area for trees, it does not give information about its distribution over the height. Therefore, a single tree was fully measured (i.e., average diameter $b_{v, i}$ and the number of branches $N_{i}$ at every meter along the height of the tree) to determine the frontal surface area distribution over the vertical. This distribution was assumed to hold for all the trees in the flume. Both measurements at breast height and the detailed sketches of the primary branches were input to determine these initial parameters and branching factors. These detailed sketches were also used as material to validate the outcome of this branching method. (See Supplementary Information for further details)

Wave dissipation model. The spectral wave model SWAN (Simulating Waves Nearshore) ${ }^{22}$ following Suzuki et al. (2012) was used to model the amount of wave dissipation by willow trees. SWAN was run in its 1D stationary mode, in a Cartesian and regular computational grid. The willow forest was modelled by accounting for 7 vertical layers of vegetation (expressed as frontal surface area), which were assumed to be uniform along the forest length. SWAN is based on the bulk wave dissipation (integrated over all wave frequencies), which depends on the incoming wave energy, relative water depth and vegetation characteristics:

$$
\left\langle\varepsilon_{v}\right\rangle=\sum_{i=1: N} \frac{1}{4 \sqrt{2 \pi}} \rho \tilde{C}_{D}\left(\frac{g k}{2 \sigma}\right)^{3} f_{i} \frac{\left(\sinh ^{3} k \alpha_{i} h-\sinh ^{3} k \alpha_{i-1} h\right)+3\left(\sinh k \alpha_{i} h-\sinh k \alpha_{i-1} h\right)}{3 k \cosh ^{3} k h} H_{s}^{3}
$$

\section{(Equation 2)}

Where $\left\langle\varepsilon_{v}\right\rangle$ is the averaged wave energy dissipation due to vegetation, $\tilde{C}_{D}$ the bulk drag coefficient, $g$ the gravitational acceleration constant, $k$ the mean wave number, $\alpha$ the portion of the water depth covered by vegetation for layer $\mathrm{i}, h$ the water depth, $H_{s}$ the significant wave height, and $\mathrm{f}_{\mathrm{i}}$ the total frontal width of vegetation per surface area for layer $i$, which is equivalent to the generally used $b_{v, i} N_{v, i}$.

A correlation was found between the bulk drag coefficient $\left(\tilde{C}_{D}\right)$ and the $\mathrm{KC}$ number ${ }^{34}$, as shown in Figure $3 \mathrm{~b}$. The $K C$ number is defined as $K C=\hat{u}_{s} T_{m} / D_{v}$, where $\hat{u}_{s}$ is the characteristic velocity, $D_{v}$ is a representative diameter for the branches of the entire tree, and $T_{m}$ is the wave period. The characteristic velocity $\left(\hat{u}_{S}\right)$ is the maximum velocity per layer integrated over the water depth based on linear wave theory. The representative diameter $\left(D_{v}\right)$ is determined for each water depth as the 
branch diameter weighted over the number of branches per vegetation layer and over the layer thickness. The Keulegan - Carpenter number $(K C)$ was determined by considering separate layers over the height. This was necessary as willow trees have complex geometries which involve diameter decay and varying branch densities. Several other references use the total width per tree/plant, which has no direct physical meaning in the sense of the original definition of the $\mathrm{KC}$ number ${ }^{34}$. Additionally, other studies show differences in flexibility and absence of extra viscous forces that influence results at a small scale.

\section{References}

1. Costanza, R. et al. The value of the world's ecosystem services and natural capital. Nature 387, 253-260 (1997).

2. Temmerman, S. et al. Ecosystem-based coastal defence in the face of global change. Nature 504, 79-83 (2013).

3. Koch, E. W. et al. Non-linearity in ecosystem services: temporal and spatial variability in coastal protection. Front. Ecol. Environ. 7, 29-37 (2009).

4. Gedan, K. B., Kirwan, M. L., Wolanski, E., Barbier, E. B. \& Silliman, B. R. The present and future role of coastal wetland vegetation in protecting shorelines: answering recent challenges to the paradigm. Clim. Change (2011) doi:DOI: 10.1007/s10584-010-0003-7.

5. Zhu, Z. et al. Historic storms and the hidden value of coastal wetlands for nature-based flood defence. Nat. Sustain. 3, (2020).

6. Shepard, C. C., Crain, C. M. \& Beck, M. W. The Protective Role of Coastal Marshes: A Systematic Review and Meta-analysis. PLoS One 6, e27374 (2011).

7. Coops, H., Boeters, R. \& Smit, H. Direct and indirect effects of wave attack on helophytes. Aquat. Bot. 41, 333-352 (1991).

8. van Wesenbeeck, B. K. et al. Coastal and riverine ecosystems as adaptive flood defenses under a changing climate. Mitig. Adapt. Strateg. Glob. Chang. 22, 1-8 (2016).

9. Dalrymple, R. A., Kirby, J. T. \& Hwang, P. A. Wave diffraction due to areas of energy dissipation. J. Waterw. Ports Coast. Eng. 110, 67-69 (1984).

10. Suzuki, T., Zijlema, M., Burger, B., Meijer, M. C. \& Narayan, S. Wave dissipation by vegetation with layer schematization in SWAN. Coast. Eng. 59, 64-71 (2012).

11. Maza, Maria, Lara, J.L., Losada, I. . Experimental analysis of wave attenuation and drag forces in a realistic fringe Rhizophora mangrove forest. Adv. Water Resour. 131, (2019).

12. Nepf, H. M. Drag, turbulence, and diffusion in flow through emergent vegetation. Water Resour. Res. 35, 479-489 (1999).

13. Wolters, M. et al. Saltmarsh erosion and restoration in south-east England: squeezing the evidence requires realignment. J. Appl. Ecol. 42, 844-851 (2005).

14. Vuik, V., Jonkman, S. N., Borsje, B. W. \& Suzuki, T. Nature-based flood protection: The efficiency of vegetated foreshores for reducing wave loads on coastal dikes. Coast. Eng. 116, 
42-56 (2016).

15. Yang, S. L., Shi, B. W., Bouma, T. J., Ysebaert, T. \& Luo, X. X. Wave Attenuation at a Salt Marsh Margin: A Case Study of an Exposed Coast on the Yangtze Estuary. Estuaries and Coasts 35, 169-182 (2012).

16. Bouma, T. J. et al. Trade-offs related to ecosystem engineering: A case study on stiffness of emerging macrophytes. Ecology 86, 2187-2199 (2005).

17. Bouma, T. J., De Vries, M. B. \& Herman, P. M. J. Comparing ecosystem engineering efficiency of two plant species with contrasting growth strategies. Ecology 91, 2696-2704 (2010).

18. Ysebaert, T. et al. Wave attenuation by two contrasting ecosystem engineering salt marsh macrophytes in the intertidal pioneer zone. in Wetlands vol. 31 (2011).

19. Granek, E. \& Ruttenberg, B. I. Changes in biotic and abiotic processes following mangrove clearing. Estuar. Coast. Shelf Sci. 80, 555-562 (2008).

20. Mazda, Y., Magi, M., Ikeda, Y., Kurokawa, T. \& Asano, T. Wave reduction in a mangrove forest dominated by Sonneratia sp. Wetl. Ecol. Manag. 14, 365-378 (2006).

21. Horstman, E. M. et al. Wave attenuation in mangroves: A quantitative approach to field observations. Coast. Eng. 94, 47-62 (2014).

22. Booij, N., Ris, R. C. \& Holthuijsen, L. H. A third-generation wave model for coastal regions: 1. Model description and validation. J. Geophys. Res. 104, 7649-7666 (1999).

23. Dalrymple, R. A., Kirby, J. T. \& Hwang, P. A. Wave diffraction due to areas of energy dissipation. J. Waterw. Ports Coast. Eng. 110, 67-69 (1984).

24. Mendez, F. J. \& Losada, I. J. An empirical model to estimate the propagation of random breaking and nonbreaking waves over vegetation fields. Coast. Eng. 51, 103-118 (2004).

25. Järvelä, J. Determination of flow resistance caused by non-submerged woody vegetation. Int. J. River Basin Manag. 2, 61-70 (2004).

26. Sumer, M. \& Fredsøe, J. BOOK REVIEW HYDRODYNAMICS AROUND CYLINDRICAL STRUCTURES, B. M. Sumer and J.Fredsøe, World Scientific, Singapore. J. Fluids Struct. 12, 221-222 (1998).

27. Mendez, F. J., Losada, I. J., Dalrymple, R. A. \& Losada, M. A. Effects of wave reflection and dissipation on wave-induced second order magnitudes. in Coastal Engineering 1998, Vols 1-3 (ed. Edge, B. L.) 537-550 (1999).

28. Jadhav, R. \& Chen, Q. Field investigation of wave dissipation over salt marsh vegetation during tropical cyclone. in (2012).

29. Anderson, M. E. \& Smith, J. M. Wave attenuation by flexible, idealized salt marsh vegetation. Coast. Eng. 83, (2014).

30. Möller, I. et al. Wave Dissipation and Transformation Over Coastal Vegetation Under Extreme Hydrodynamic Loading. HYDRALAB IV Jt. user Meet. 1-6 (2014).

31. Jadhav, R. S., Chen, Q. \& Smith, J. M. Spectral distribution of wave energy dissipation by salt marsh vegetation. Coast. Eng. 77, (2013). 
32. Ozeren, Y., Wren, D. G. \& Wu, W. Experimental Investigation of Wave Attenuation through Model and Live Vegetation. J. Waterw. Port, Coastal, Ocean Eng. 140, 04014019 (2014).

33. He, F., Chen, J. \& Jiang, C. Surface wave attenuation by vegetation with the stem, root and canopy. Coast. Eng. 152, (2019).

34. Keulegan, G. H. \& Carpenter, L. H. Forces on cylinders and plates in an oscillating fluid. J. Res. Natl. Bur. Stand. (1934). 60, (1958).

35. Sutton-Grier, A. E., Wowk, K. \& Bamford, H. Future of our coasts: The potential for natural and hybrid infrastructure to enhance the resilience of our coastal communities, economies and ecosystems. Environ. Sci. Policy 51, 137-148 (2015).

36. Cheong, S. M. et al. Coastal adaptation with ecological engineering. Nat. Clim. Chang. 3, 787791 (2013).

37. Borsje, B. W. et al. How ecological engineering can serve in coastal protection. Ecol. Eng. 37, 113-122 (2011).

38. Wuytack, T. et al. The potential of biomonitoring of air quality using leaf characteristics of white willow (Salix alba L.). Environ. Monit. Assess. 171, 197-204 (2010).

39. Jacobsen, N. G., Bakker, W., Uijttewaal, W. S. J. \& Uittenbogaard, R. Experimental investigation of the wave-induced motion of and force distribution along a flexible stem. $J$. Fluid Mech. 1036-1069 (2019) doi:10.1017/jfm.2019.739.

\section{Statement on plant materials}

Experiments in this research were executed with cultivated willow species (Salix alba) of 15 years old that were obtained from a Dutch private site. Trees were replaced with new younger trees. Salix alba does not occur on the list of threatened species for the Netherlands and is labeled as stable.

\section{Acknowledgements}

We thank van Oord, Boskalis, Rijkswaterstaat, World Wildlife Fund, Stowa, and VP Delta for their contributions to this research. Part of this study was done within the research program WOODY, number 17194, which is financed by the Dutch Research Council (NWO) via the Open Technology Program. We thank Hassan Niazi for helping with the measurements. We thank everyone that contributed to this research through crowdfunding. Without all of you this work on nature-based solutions would not be possible. 


\section{Supplementary information}

Experimental set-up and measurements
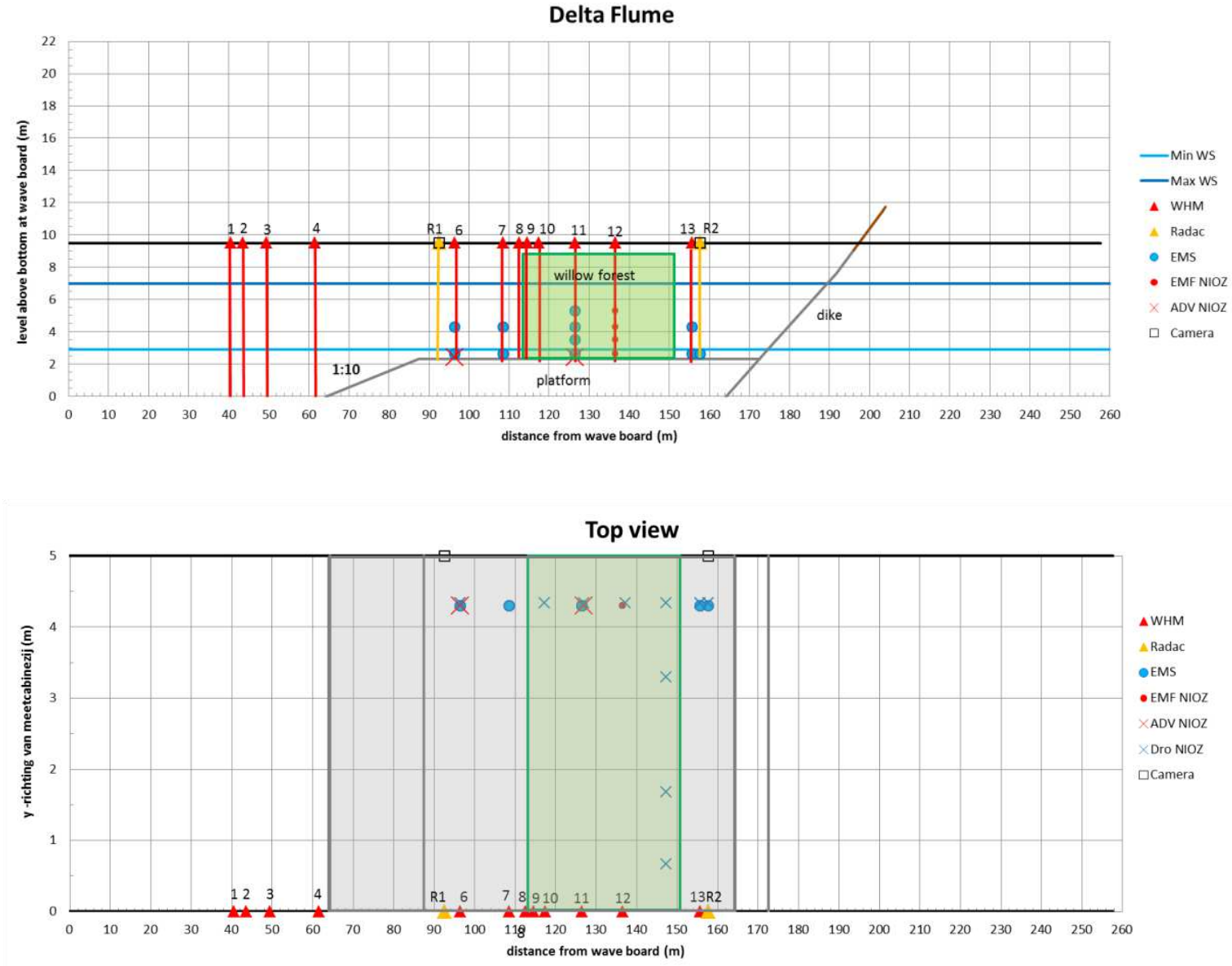

Figure S1. Model setup and instrumentation; Upper panel: Side view, Lower panel: Top view Abbreviations: $W H M=$ wave gauge (resistance type), Radac = radar wave gauge, $D R O=$ pressure sensor, $E M S=$ electromagnetic velocity sensor, EMF = electromagnetic velocity sensor (provided by NIOZ), ADV = Acoustic Doppler Velocity meter (provided by NIOZ) 


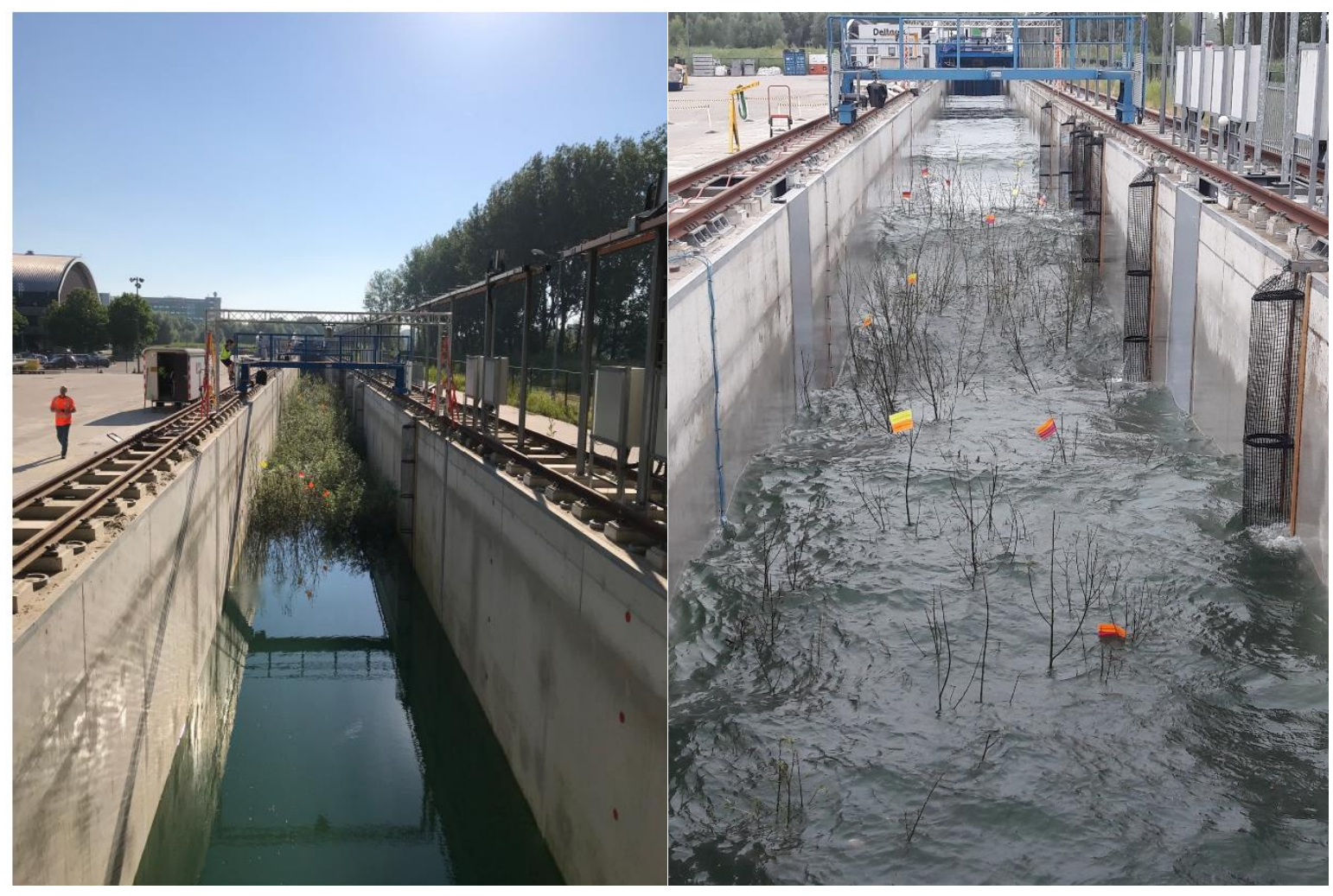

Figure S2. Left: Trees with leaves in flume with $3 \mathrm{~m}$ water depth in forest. Right: Trees without leaves with 4,5 meter water level.

Table S2. Hydrodynamic conditions per test measured at the beginning of the foreshore by RADAC1 and velocity meter WHM06. $H_{m 0,0}$ is the significant wave height in front of the forest; $H_{\max }$ the maximum wave height in front of the forest; $T_{p}$ the peak period; $h$ the water depth in the willow forest; $s_{o p}$ the wave steepness defined as the ratio between $H_{m 0}$ and the wave length computed with the mean period of the sea state in deep waters; $H_{m 0,1 \text {, willows }}$ the wave height measured behind

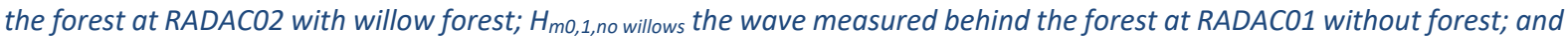
$C d$ the bulk drag coefficient as result of SWAN modelling. For the vegetation treatment conditions (series) see Table 1.

\begin{tabular}{llllllllll}
\hline Test & Series & $\begin{array}{l}\mathrm{H}_{\mathrm{m} 0,0} \\
{[\mathrm{~m}]}\end{array}$ & $\begin{array}{l}\mathrm{H}_{\max } \\
{[\mathrm{m}]}\end{array}$ & $\begin{array}{l}\mathrm{Tp}_{\mathrm{p}} \\
{[\mathrm{s}]}\end{array}$ & $\begin{array}{l}\mathrm{h} \\
\text { Water } \\
\text { depth } \\
{[\mathrm{m}]}\end{array}$ & $\begin{array}{l}\mathrm{S}_{\mathrm{op}} \\
\text { Wave } \\
\text { steepness } \\
{[-]}\end{array}$ & $\begin{array}{l}\mathrm{H}_{\mathrm{m} 0,1,} \\
\text { willows } \\
{[\mathrm{m}]}\end{array}$ & $\begin{array}{l}\mathrm{H}_{\mathrm{m} 0,1,} \\
\text { nowillows } \\
{[\mathrm{m}]}\end{array}$ & $\begin{array}{l}\mathrm{Cd} \\
{[-]}\end{array}$ \\
\hline 5 & 2 & 0.43 & 0.85 & 2.84 & 3 & 0.04 & 0.37 & 0.41 & 2.24 \\
\hline 6 & 2 & 0.97 & 1.56 & 3.92 & 3 & 0.05 & 0.68 & 0.87 & 1.19 \\
\hline 7 & 2 & 0.47 & 0.79 & 3.93 & 3 & 0.03 & 0.36 & 0.44 & 1.87 \\
\hline 8 & 2 & 0.95 & 1.75 & 5.57 & 3 & 0.03 & 0.75 & 0.96 & 1.17 \\
\hline 13 & 3 & 0.44 & 0.80 & 2.84 & 3 & 0.05 & 0.33 & 0.41 & 1.64 \\
\hline 14 & 3 & 0.95 & 1.64 & 3.57 & 3 & 0.06 & 0.72 & 0.87 & 1.02 \\
\hline 15 & 3 & 0.45 & 0.79 & 3.93 & 3 & 0.03 & 0.37 & 0.44 & 1.84 \\
\hline 16 & 3 & 0.97 & 1.76 & 5.57 & 3 & 0.03 & 0.77 & 0.96 & 1.07 \\
\hline 21 & 3 & 1.40 & 2.19 & 4.80 & 4.5 & 0.05 & 1.26 & 1.39 & 0.48 \\
\hline 22 & 3 & 1.41 & 2.45 & 6.85 & 4.5 & 0.03 & 1.36 & 1.50 & 0.62 \\
\hline 23 & 4 & 0.43 & 0.79 & 2.84 & 3 & 0.05 & 0.36 & 0.41 & 1.8 \\
\hline 24 & 4 & 0.93 & 1.59 & 3.57 & 3 & 0.06 & 0.77 & 0.87 & 1.11 \\
\hline 25 & 4 & 0.43 & 0.78 & 3.93 & 3 & 0.02 & 0.39 & 0.44 & 1.73 \\
\hline
\end{tabular}




\begin{tabular}{llllllllll}
\hline 26 & 4 & 0.97 & 1.73 & 5.57 & 3 & 0.03 & 0.83 & 0.96 & 1.26 \\
\hline 29 & 4 & 1.40 & 2.23 & 4.80 & 4.5 & 0.05 & 1.30 & 1.39 & 0.58 \\
\hline 30 & 4 & 1.44 & 2.52 & 6.85 & 4.5 & 0.03 & 1.41 & 1.50 & 0.77 \\
\hline 33 & 5 & 0.44 & 0.76 & 2.84 & 3 & 0.05 & - & 0.41 & 11.9 \\
\hline 34 & 5 & 0.94 & 1.52 & 3.92 & 3 & 0.05 & - & 0.87 & 11.9 \\
\hline 35 & 5 & 0.46 & 0.78 & 3.93 & 3 & 0.03 & - & 0.44 & 11.9 \\
\hline 36 & 5 & 0.96 & 1.67 & 5.57 & 3 & 0.03 & - & 0.96 & 11.9 \\
\hline 37 & 5 & 1.40 & 2.19 & 4.80 & 4.5 & 0.05 & - & 1.39 & 11.9 \\
\hline 38 & 5 & 1.43 & 2.51 & 6.85 & 4.5 & 0.03 & - & 1.50 & 11.9 \\
\hline
\end{tabular}

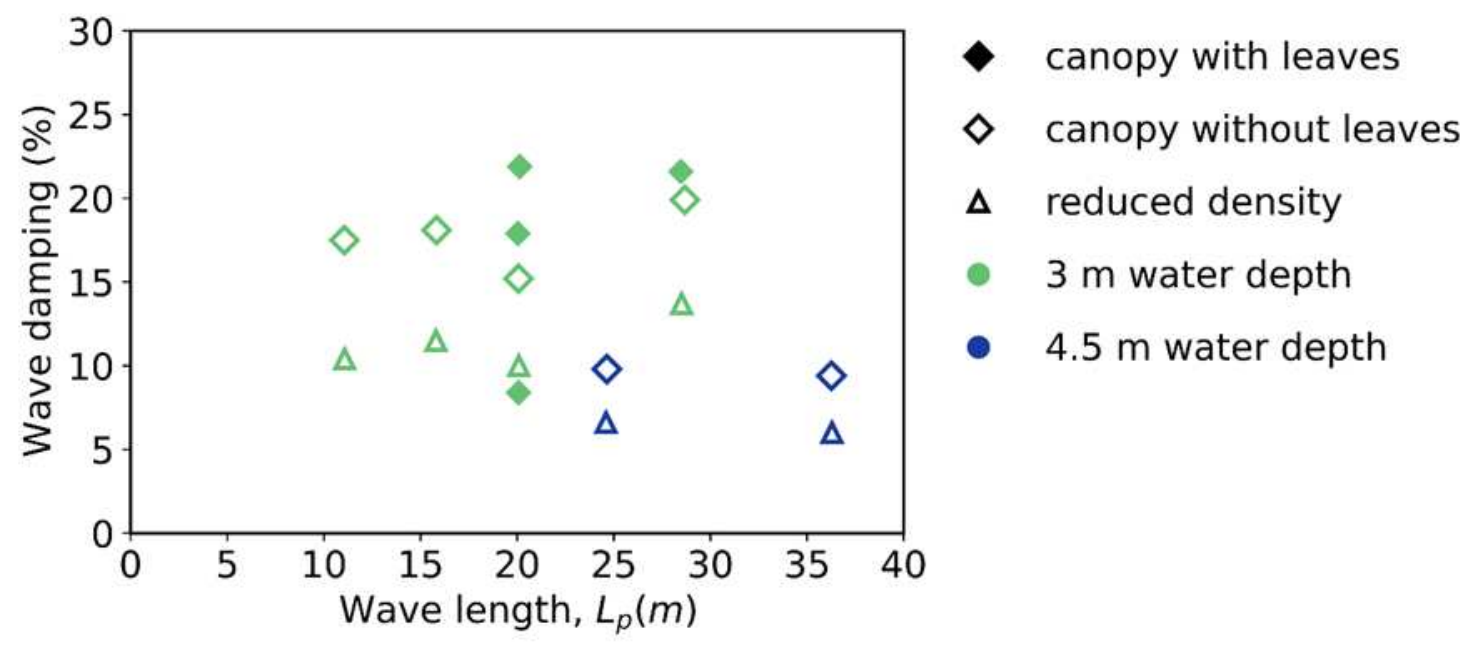

Figure S3. Relation between measured wave damping ([incoming-transmitted]/incoming $x$ 100) and incoming significant wave height at the start of the forest (left panel), or incoming wave length (right panel). Markers represent the different treatments applied in the willow forest and the filling of the markers show tested water levels (3 metres and 4.5 metres).

Determination of frontal surface area distribution

According to the branching ordering scheme, based on the work of Jarvela (2004), the lowest order begins at the tip of the branches and approaches the highest order (usually the trunk). It requires the following initial parameters, namely: $d_{\text {min }}, N_{\text {high }}, d_{\text {high }}, L_{\text {high }}$, where $\mathrm{d}_{\min }$ is the diameter of the smallest branch, $N_{h i g h}, d_{\text {high }}$ and $L_{\text {high }}$ are respectively the number of branches, the diameter and the length of the highest order branches. A description of the steps can be found in the paper by Jarvela (2004), applied on branches of natural willows. 


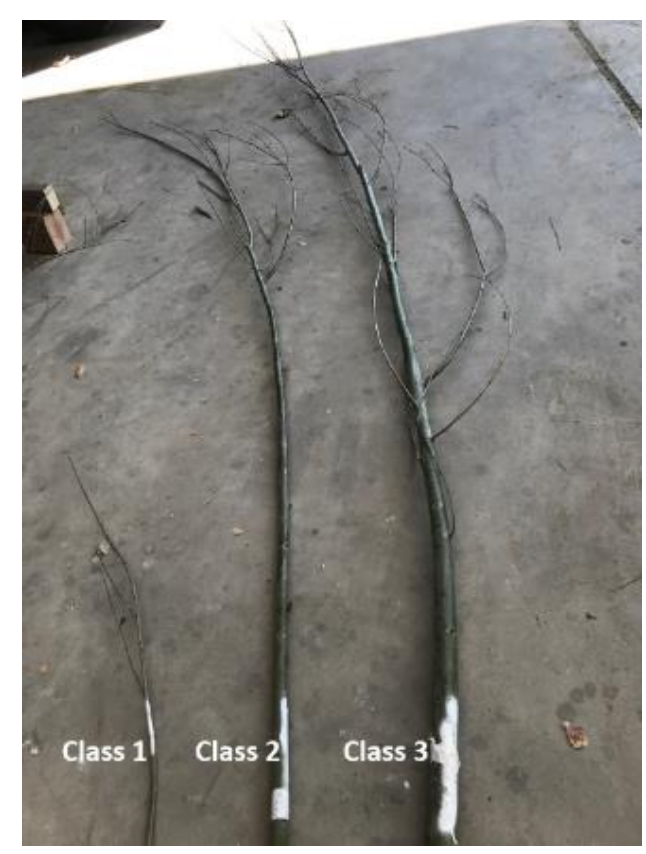

Figure S4. Example of the 3 branch classes

Table S3. Initial parameters for each branch class for one tree.

\begin{tabular}{lllll}
\hline & $\mathrm{N}_{\mathrm{m}, \text { high }}(\mathrm{m})$ & $\mathrm{d}_{\text {high }}(\mathrm{m})$ & $L_{\text {high }}(\mathrm{m})$ & $\mathrm{d}_{\min }(\mathrm{m})$ \\
\hline class 1 $(\mathrm{d}>50 \mathrm{~mm})$ & 10 & 0.061 & 3.85 & 0.003 \\
class 2 $(20<\mathrm{d}<50 \mathrm{~mm})$ & 19 & 0.037 & 2.52 & 0.003 \\
class 3 $(\mathrm{d}<20 \mathrm{~mm})$ & 29 & 0.013 & 0.82 & 0.003 \\
\hline
\end{tabular}

In this study the values and method were adjusted to account for the branching structure of pollard willows. Firstly, the initial parameters were determined for each branch class as shown in Table S3.

Table S4. Branching factors

\begin{tabular}{lll}
\hline & $(\boldsymbol{m}-\mathbf{2})^{\text {th }}$ to $(\boldsymbol{m}-\mathbf{1})^{\text {th }}$ order & $\mathbf{( m - 1}^{\text {th }}$ to $\boldsymbol{~}^{\text {th }}$ order \\
\hline $\mathbf{R}_{\mathbf{b}}$ & 4.19 & 10.56 \\
$\mathbf{R}_{\mathbf{d}}$ & 1.71 & 6.26 \\
$\mathbf{R}_{\mathrm{L}}$ & 1.44 & 4.50 \\
\hline
\end{tabular}

Secondly, instead of averaging the branching factors over all the orders, we maintained separate values for them as shown in Table S4.

Table S5. An example for all the branches of class one for a single tree in the flume

\begin{tabular}{lllll}
\hline & $\mathbf{N}$ (units) & $\mathbf{d}(\mathbf{m})$ & $\mathbf{L}(\mathbf{m})$ & Frontal area $\left(\mathbf{m}^{2}\right)$ \\
\hline Branch- order & & & & \\
M, primary branch & 10 & 0.061 & 3.85 & 2.35 \\
m-1 & 105 & 0.009 & 0.86 & 0.88
\end{tabular}




\begin{tabular}{lllll}
$\mathrm{m}-2$ & 444.2 & 0.005 & 0.6 & 1.55 \\
$\mathrm{~m}-3$, smallest branch & 1852.67 & $\mathbf{0 . 0 0 3}$ & 0.41 & 2.56 \\
\hline Frontal area, total & & & & 7.29 \\
\hline
\end{tabular}

The branching factors $\left(R_{B}, R_{D}, R_{L}\right)$ were applied starting at the highest order branches (in this case, the primary branches extending from the trunk), to calculate the diameters (d), number of branches (N) and length (L) of the subsequent order branches till the lowest order branch with a diameter of $d_{\min }=3 \mathrm{~mm}$. The total frontal surface area for each branch class is calculated by $N^{*} d^{*} L$ and taking the sum of the frontal area per order, an example for branches of class 1 is shown in Table S5. By repeating the same procedure for the other branch classes, and taking the sum of these areas, the total frontal surface area of one tree is determined (excluding the frontal area of the trunk). This method assumes linear decay of frontal area over the height and uses cylinder shapes for the branches in each order to calculate the frontal surface area. Therefore, we applied a factor of 0.5 to the frontal area results per order, to account for cone shapes instead of cylinders. Lastly, we used measurements from a single tree to determine the distribution of the total frontal surface area over the height.

\section{Determination of KC}

The KC number or period parameter defines the ratio of the distance traversed by a fluid particle during half a wave period to the diameter of the cylinder, according to the definition by Keulegan and Carpenter (1958). The table below shows an overview of the test conditions and ways in determining the $\mathrm{KC}$ number, used in some reference studies, indicating possible explanations for deviations seen in Figure 3b. For instance, other references use the total width per tree/plant, which has no direct physical meaning in the sense of the original definition of the $\mathrm{KC}$ number.

Table S6. A comparison of $\mathrm{Cd}-\mathrm{KC}$ relations between different studies.

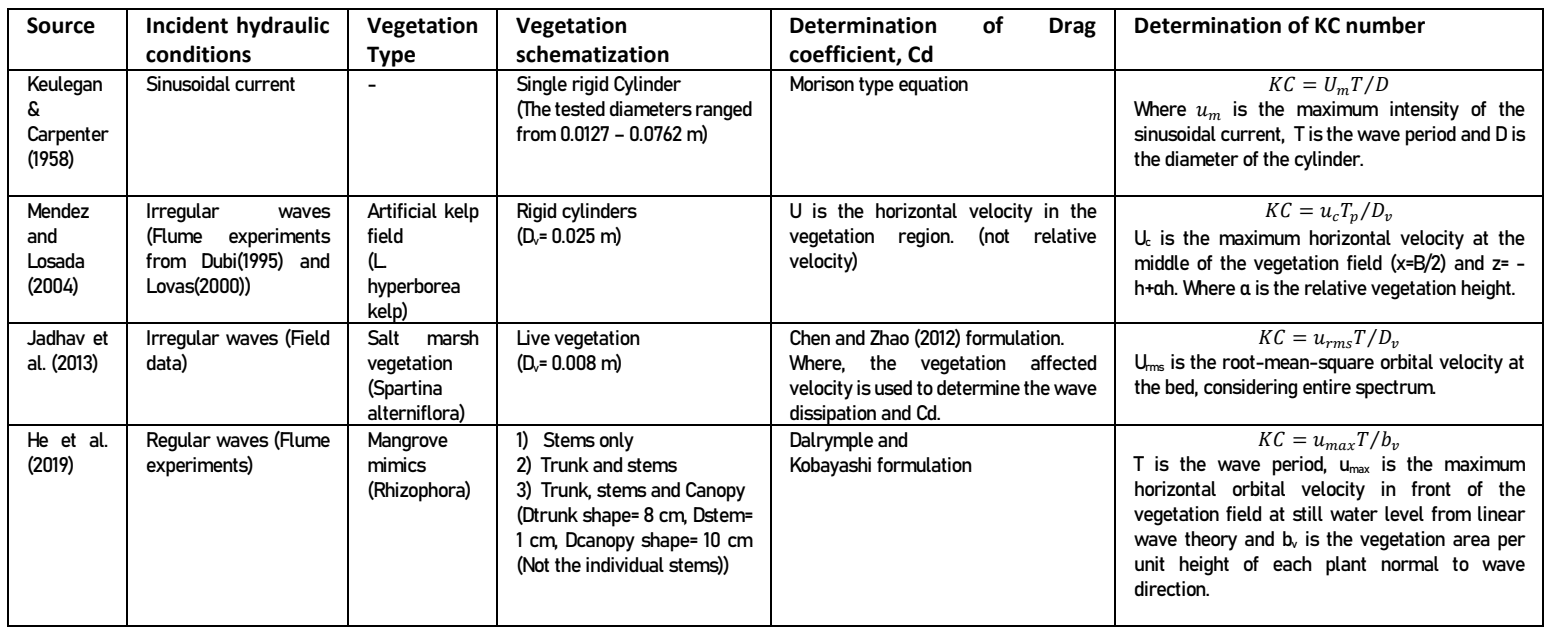


The Length ratio $\left(\mathrm{L}=\frac{l . \omega}{u_{w}}, u_{w}=\right.$ characteristic velocity; $\omega=$ the cyclic frequency; and $l=$ branch length $)$ and the Cauchy number ( $\mathrm{Ca}=\frac{\rho \cdot D \cdot u_{w .}^{2} l^{3}}{E I}, \rho=$ the fluid density; $\mathrm{D}=$ diameter of the branch; $\mathrm{E}=$ the Youngs' Modulus; and $I=$ the second moment of inertia ) are shown to be important non-dimensional parameters to describe the motion of flexible stems ${ }^{39}$. According to Jacobsen et al. (2019), a stem is considered stiff if the Inertia-to-stiffness ratio $(C a . L / K C)<0.5$. For the present experiments, this ratio ranges between 0.1 - 45 considering a primary willow branch without side branches, where the lower values correspond to the tests with water depths of $3 \mathrm{~m}$ and the higher values to the tests with water depths of $4.5 \mathrm{~m}$. This suggests relatively stiff behavior for the branches during the tests with low water levels and an increase of branch motion during the tests with higher water levels. The branch-density of the primary branches is low (about $14.4 \mathrm{~m}^{-2}$ ), which gives a mean distance between branches of, roughly, 15 times their diameter. This means that the influence of the branches on the $\tilde{C}_{D}$ due to blockage is limited, in the order of $10 \%{ }^{12}$. The Reynolds number for the largest waves based on maximum orbital motion, the significant wave height, and the largest branch diameter class was $10^{5}$. This means that even though the scale is large, the boundary layer around the branches is still laminar, such that the drag crisis, a fluid dynamics phenomenon which can reduce the drag coefficient and thereby the energy dissipation by a factor of 4 , was mostly not reached. The drag on the stem is expected to have reached the drag crisis for a small percentage of the most extreme waves.

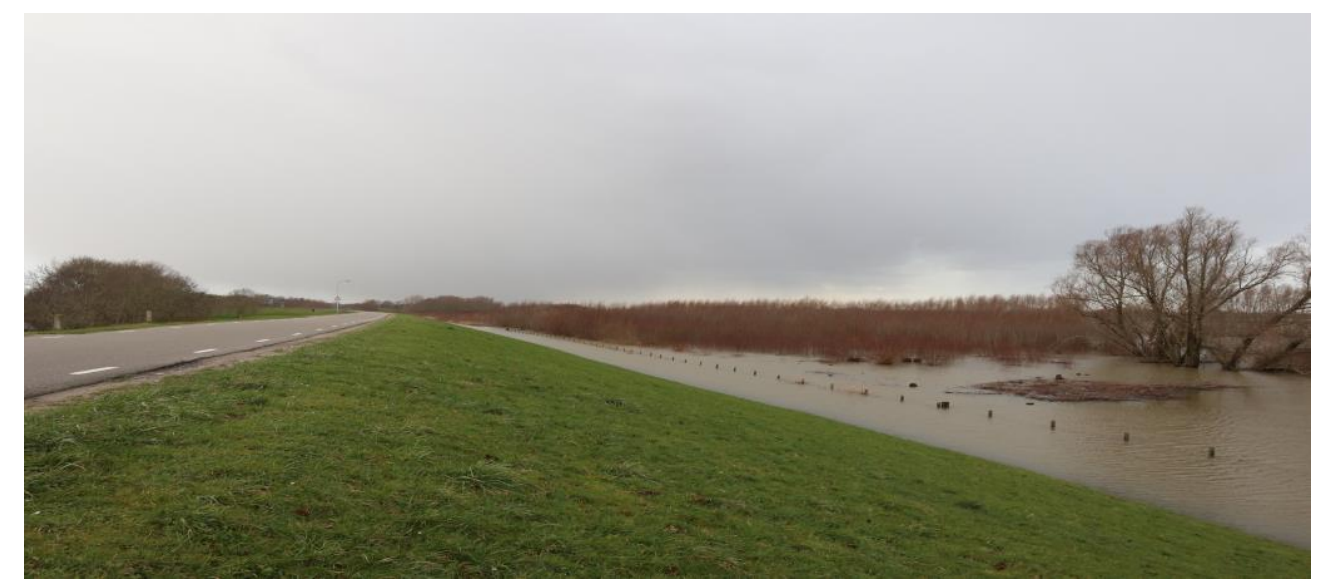

Figure S5. Willows in front of a levee in a Dutch floodplain 


\section{Figures}

A
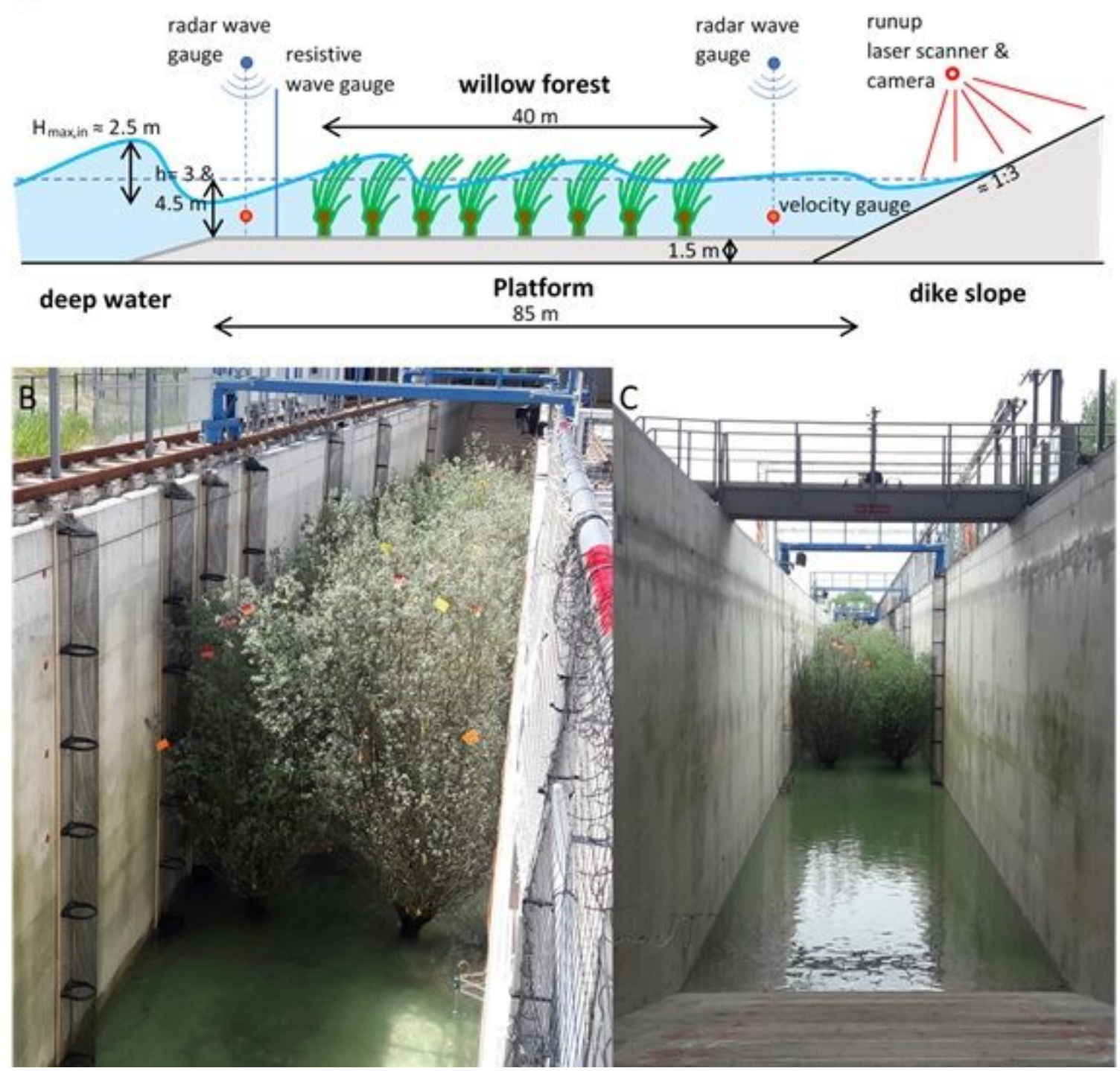

Figure 1

A. Setup of experiments inside the Delta Flume with the most important instruments, where WHM = wave gauge (resistance type), Radac = nonintrusive radar wave gauge, EMS = electromagnetic velocity sensor, B. Front top view of willow forest, C. view from the back slope. 

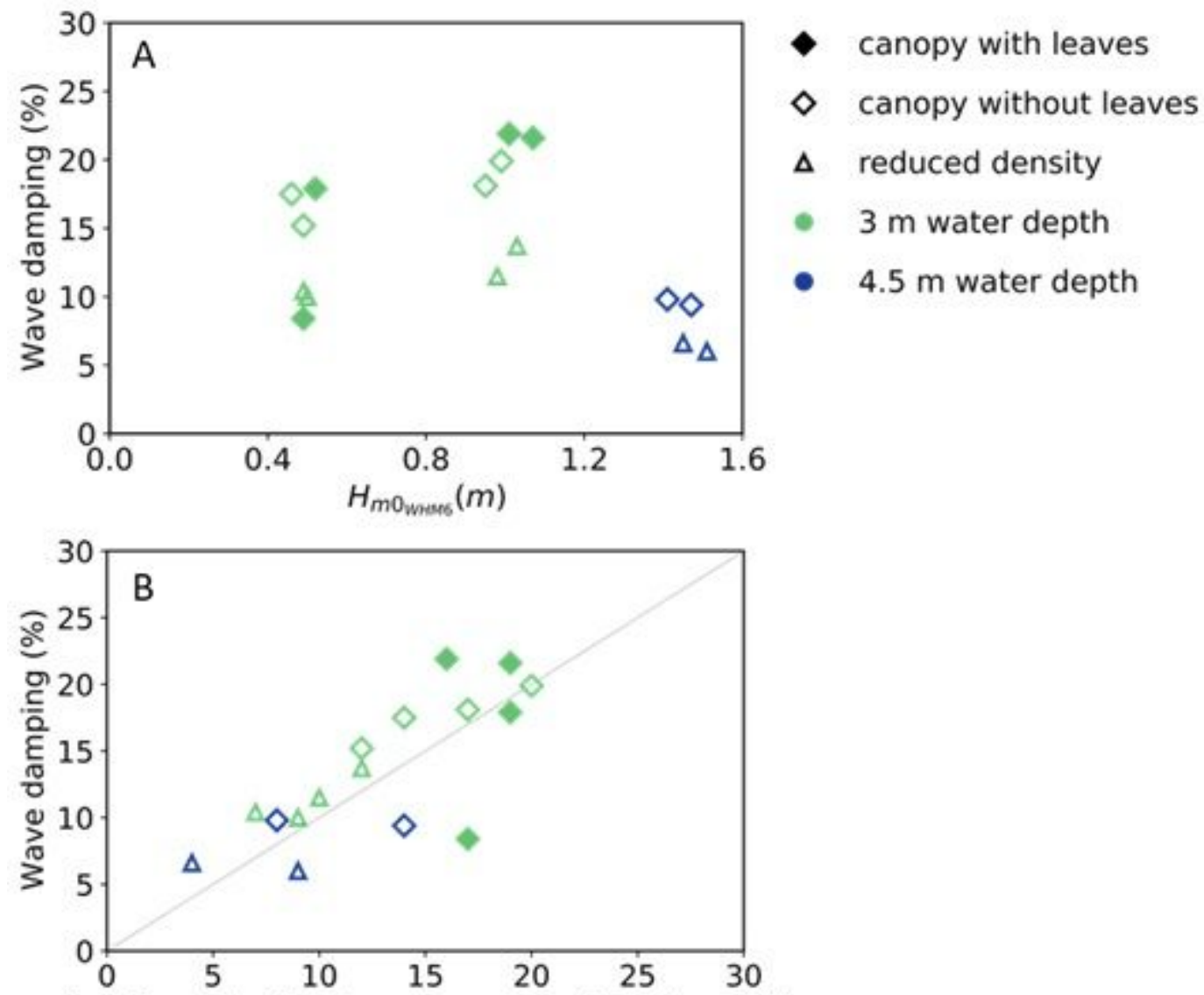

Relative reduction in wave run-up, $\Delta z_{2 \%} / z_{2 \%}(\%)$

\section{Figure 2}

A. Relation between measured wave attenuation (\%) and incoming significant wave height $(\mathrm{Hm} 0)$ at WHM6. Markers represent the different willow forest configurations $(2=$ with leaves, $3=$ without leaves, 4 $=$ reduced density branches), and the colors show water levels (green $=3 \mathrm{~m}$ and blue $=4.5 \mathrm{~m}$ ), B. Relation between relative reduction in wave run-up on the slope and the wave attenuation through the forest. 


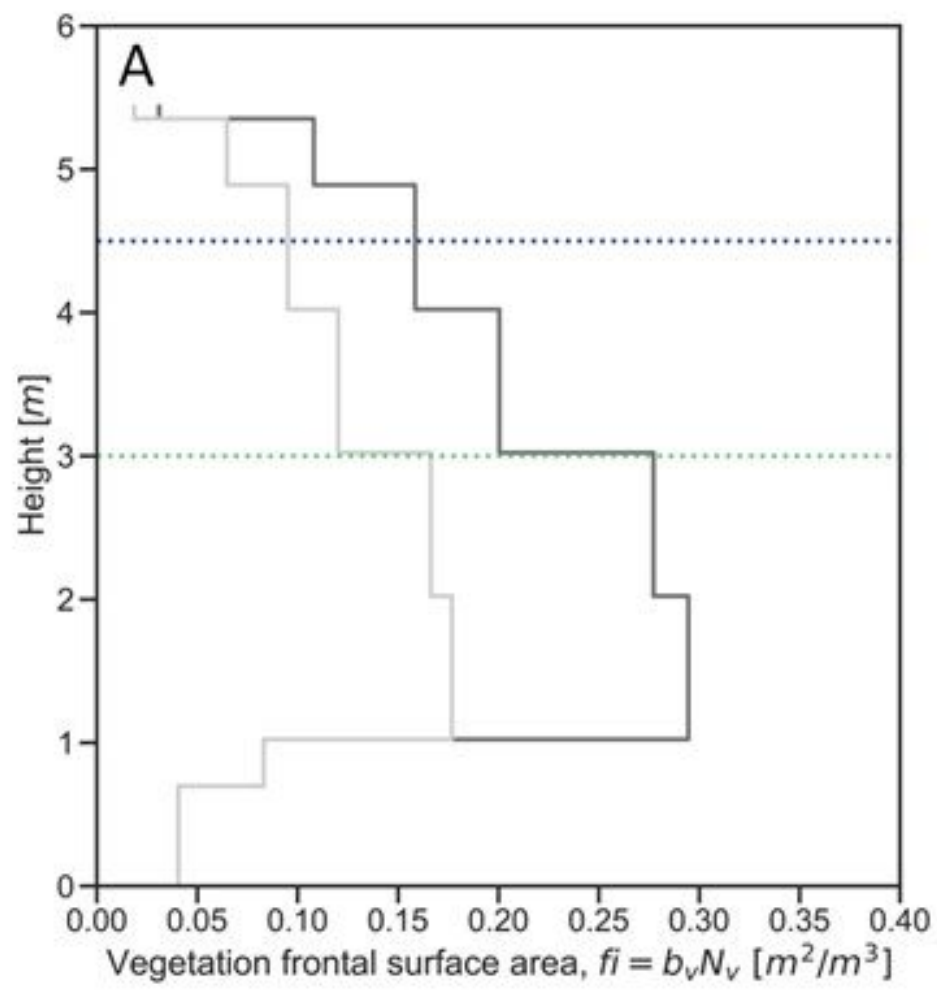

canopy with leaves,

without leaves

reduced density

$3 \mathrm{~m}$ water depth

$4.5 \mathrm{~m}$ water depth

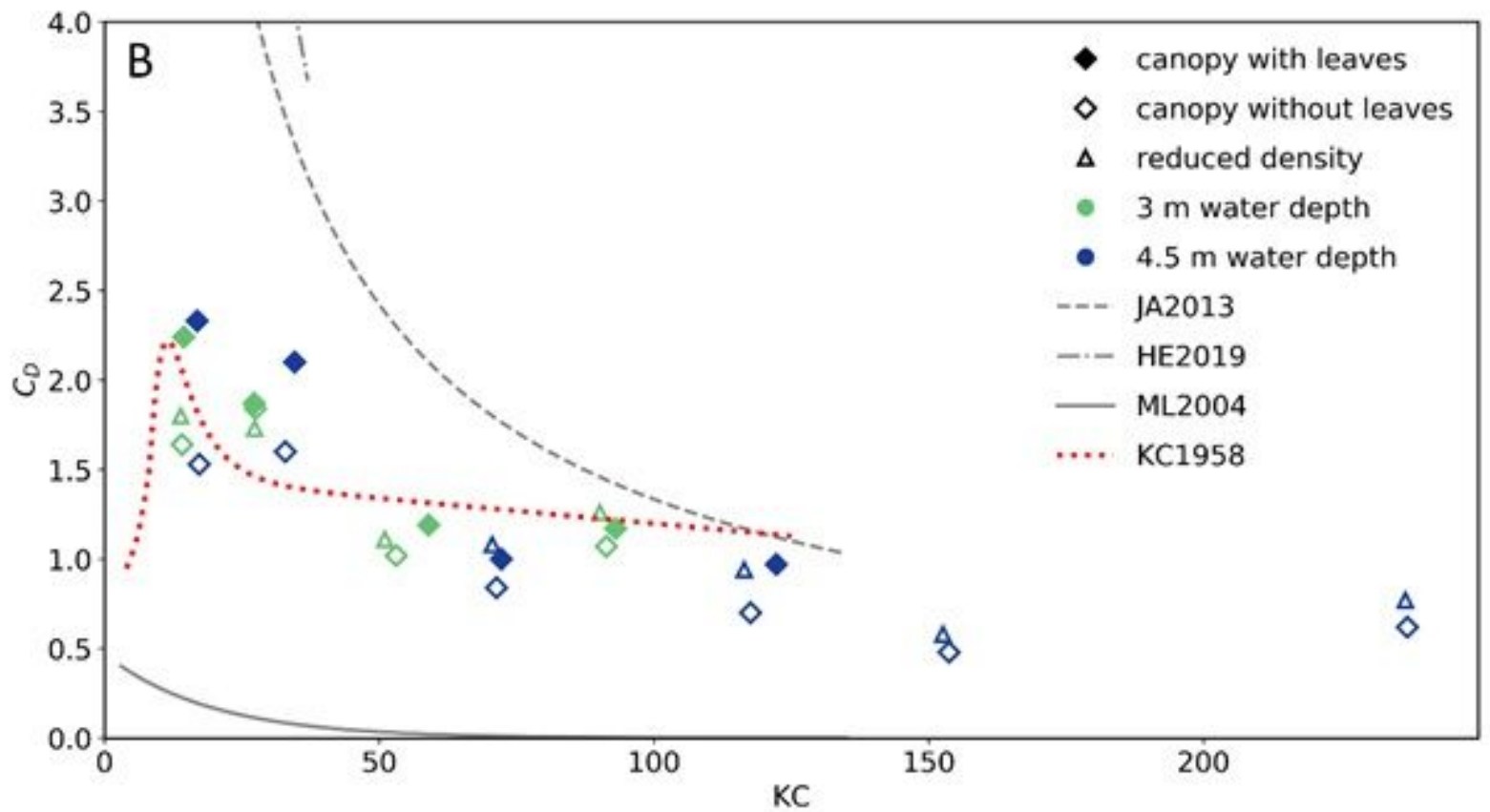

Figure 3

A. Frontal surface area of willow schematization (based in biomass schematization) for test series with full biomass with and without leaves ( 2 and 3 ) and with half of the branches removed (4), B. The KC-CD relation for all test series $(2+3+4)$ and the comparison with the relations by He et al. (2019) (HE2019), Jadhav et al. (2013) (JA2013), Mendez and Losada (2004) (ML2004), Keulegan and Carpenter (1958) (KC1958). Steady flow would be a straight line equaling $\mathrm{Cd}=1.2$. 


\section{Supplementary Files}

This is a list of supplementary files associated with this preprint. Click to download.

- SupplementaryInformation.pdf 